\title{
Psicoprofilaxis obstétrica. La realidad actual
}

\author{
Obstetric psychoprophylaxis. The current reality
}

\author{
Alejandra Martínez Schulte, ${ }^{*}$ Samuel Karchmer Krivitzky*
}

Citar como: Martínez SA, Karchmer KS. Psicoprofilaxis obstétrica. La realidad actual. Acta Med Grupo Angeles. 2021; 19 (3): 361-367. https://dx.doi.org/10.35366/101728

\section{Resumen}

Los avances científicos y tecnológicos en las últimas dos décadas han permitido el desarrollo y la aplicación de varias estrategias para iniciar, acelerar y regular el proceso fisiológico del parto, siempre intentando mejorar los resultados perinatales. Sin embargo, a pesar de todos los avances mencionados, el concepto de trabajo "normal" no se ha estandarizado. La psicoprofilaxis obstétrica es un tipo de trabajo de parto que las mujeres pueden elegir libremente, si la historia médica y obstétrica lo permiten, y la paciente y su pareja reciben la capacitación adecuada durante el embarazo. En nuestro país, varios centros privados ofrecen trabajo psicoprofilácti$\mathrm{co}$, desafortunadamente esta opción no es tan común en las instituciones públicas. El objetivo de este artículo es revisar los conceptos actuales sobre psicoprofilaxis obstétrica y servir como una herramienta de apoyo para el desarrollo de nuevos programas en hospitales públicos y privados.

Palabras clave: Parto, psicoprofilaxis obstétrica, realidad actual, conceptos.

\section{INTRODUCCIÓN}

El término "parto psicoprofiláctico" a veces se usa para describir un método de parto en el que se minimiza la intervención médica para el tratamiento del dolor durante el mismo. Existen varios métodos de parto psicoprofiláctico disponibles, los cuales surgen de los diversos enfoques que se desarrollaron a través de su historia. ${ }^{1}$

* Servicio de Ginecología y Obstetricia, Hospital Ángeles Lomas. México.

Correspondencia:

Alejandra Martínez Schulte

Correo electrónico: ale.schulte97@gmail.com

Aceptado: 01-05-2020.

www.medigraphic.com/actamedica

\section{Abstract}

Scientific and technological advances in the past two decades have allowed the development and application of several strategies to initiate, accelerate and regulate the physiological process of labor, always attempting to improve perinatal results. However, despite all said advances the concept of "normal" labor has not been standardized. Obstetric psychoprophylaxis is a type of labor women can freely choose, if medical and obstetrical history allow it, and the patient as well as her partner receive adequate training throughout the pregnancy period. In our country several private centers offer psychoprophylactic labor, unfortunately this option is not as common in public institutions. The objective of this paper is to review current concepts on obstetric psychoprophylaxis, and serve as a support tool for the development of new programs both private and public hospitals.

Keywords: Childbirth, obstetric psychoprophylaxis, current reality, concepts.

En el siglo XX, en 1930, el obstetra inglés Grantly DickRead acuñó el concepto de parto natural para referirse a una atención sin anestesia e intervenciones menores. En la misma década, en la ex Unión Soviética, los médicos Nikolaiev y Platonov comenzaron a aplicar a las mujeres en trabajo de parto las técnicas del fisiólogo Iván Pavlov, considerando que las mujeres estaban condicionadas por su cultura a tener dolor durante el parto y vivirlo como una experiencia traumática; por eso había que "limpiar la mente" con un reacondicionamiento, conocido después como psicoprofilaxis. Al inicio de los años 40 , se describieron las bases fisiológicas de cómo el temor interfiere con el trabajo de parto, al aumentar el dolor y ansiedad. ${ }^{1-3}$ Con estos elementos de base, el médico francés Fernand Lamaze perfeccionó el método psicoprofiláctico como una alternativa a la intervención médica durante el parto, considerando la respiración y las técnicas de la relajación para mejorar la oxigenación y disminuir el dolor. El método de Lamaze ganó renombre en los Estados Unidos después 
de que Marjorie Karmel escribiera sobre su experiencia en 1959 en su libro Thank You, Dr Lamaze. ${ }^{4-6}$ En ese mismo año se creó la Sociedad Americana de Psicoprofilaxis en Obstetricia (ASPO), y en 1960 la Asociación Internacional de Educadoras para el Parto (ICEA). En 1976 se publicó el libro Por un nacimiento sin violencia, de Frederick Leboyer, que revolucionó la obstetricia y enriqueció el modelo de atención psicoprofiláctica al describir por primera vez el proceso del parto desde el punto de vista de la persona próxima a nacer como principal protagonista: la mujer da a luz, el bebé nace. Leboyer reflexiona sobre la violencia intrínseca del nacimiento en el modelo convencional y, para mitigarla, ofrece una serie de claves para un nacimiento sin violencia. En los años 80, se profundizó en la importancia del apego en la salud física y emocional del binomio madrehijo con publicaciones como las de los pediatras Marshall Klaus y John Kenell, del gineco-obstetra e investigador francés Michel Odent y del médico David Chamberlain, con descubrimientos científicos sobre la personalidad de la/el recién nacido y su relación con el tipo de atención en el parto. Con estos aportes, se estudió desde el enfoque pediátrico la experiencia de separación de la madre, el proceso de respiración de manera natural, el impacto de los cambios de temperatura, la tranquilidad de la madre, el contacto físico piel a piel, la importancia de la escucha de los latidos cardiacos de la madre por el/la bebé, y los aportes de la lactancia temprana y del retraso en el corte del cordón umbilical. Previamente, el tema de la lactancia materna había empezado también a tomar relevancia con la fundación de la Liga de la Leche Internacional, en 1956, por un grupo de mujeres de Illinois, Estados Unidos, que habían amamantado a sus hijas/os para ayudar a quienes por diferentes razones tenían dificultades o dudas sobre cómo amamantar a sus bebés. En 1964 se abrieron grupos en México, y en la actualidad la Liga se extiende a más de 65 países, atendiendo a más de 300,000 mujeres al mes. En la década de los 90, se unificó el término "parto humanizado" para referirse al modelo de atención que hace énfasis en el protagonismo de las mujeres, dando prioridad a la toma de decisiones de las mujeres, la posición vertical del parto, el apego inmediato y la lactancia materna. Otros términos utilizados en América latina y Europa son "nacimiento humanizado", "parto respetado" y "parto digno". 7

\section{DEFINICIONES}

La definición de Orts (1983) sobre la psicoprofilaxis aún tiene vigencia: "preparación física y psicológica de la pareja, para lograr el nacimiento de la forma más natural y más segura a través de un parto consciente y activo y como consecuencia sin sufrimiento". Hoy implica también la preparación de sus parejas (Stoppard, 2004). Así, incluye actividades preparatorias para las mujeres, como ejercicios obstétricos, relajación y manejo de la respiración; y para los hombres, dinámicas como el manejo de su "embarazo" y la preparación para cuando nazca el bebé (Sapién, 2006). ${ }^{7}$

La psicoprofilaxis a su vez se divide de acuerdo con la etapa de preparación:

Psicoprofilaxis prenatal: es la preparación integral, teórica, física y psicoafectiva que se brinda durante la gestación. El mejor momento para iniciarla es entre el quinto $y$ sexto mes prenatal; sin embargo, hay que tener presente que nunca es tarde para realizarla.

Psicoprofilaxis intranatal: es la preparación integral, teórica, física y psicoafectiva que se brinda durante el trabajo de parto. Por el periodo en que se realiza, priorizará la información hacia una respuesta adecuada durante la labor.

Psicoprofilaxis postnatal: es la preparación integral, teórica, física y psicoafectiva que se brinda después del parto. Por lo general se da durante el periodo de puerperio mediato, pudiendo ampliarse hasta los primeros meses del periodo de lactancia exclusiva. Su finalidad es lograr la recuperación y reincorporación óptima de la madre, promoviendo cuidados en su salud sexual y reproductiva.

Psicoprofilaxis de emergencia: es la preparación brindada a la gestante faltando pocos días para la fecha probable de parto, o la que se ofrece durante su labor de parto. Es importante la adaptación de un programa o sesión intensiva teniendo en cuenta las prioridades. Busca esencialmente la tranquilidad, colaboración y participación de la paciente para un mejor control emocional.

Psicoprofilaxis en situaciones especiales: preparación prenatal integral que se da a los casos cuya gestación no reúne las condiciones normales y/o ideales, por lo que, de acuerdo con cada situación especial, requerirá una atención basada en las necesidades específicas de cada madre. ${ }^{7-9}$

De acuerdo con lo antes mencionado, existen distintos tipos de preparación para el parto psicoprofiláctico:

Preparación física: entrenamiento que se brinda a la gestante de acuerdo con sus condiciones generales y obstétricas para que obtenga beneficios corporales y mentales. Puede ser a través de técnicas de actividad respiratoria, de relajación neuromuscular, posiciones antálgicas y/o masajes localizados, entre otros. ${ }^{3,4}$

Preparación psicoafectiva: apoyo emocional que se brinda a la paciente y su pareja, permitiéndole disfrutar la llegada del bebé sin temores o ideas negativas, 

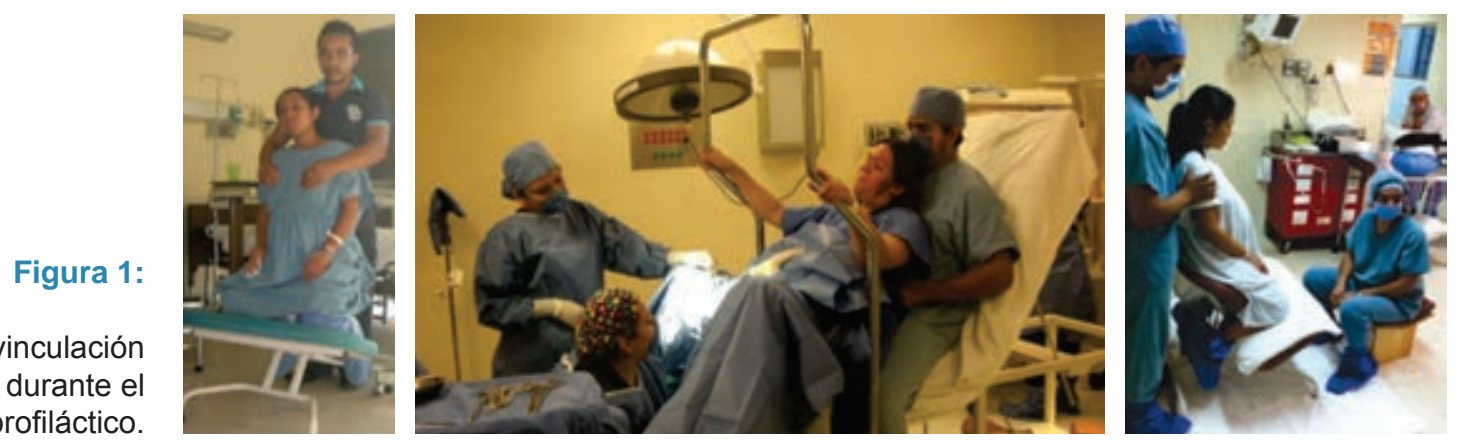

Técnicas de vinculación
en la pareja durante el parto psicoprofiláctico.

logrando en ellos tranquilidad, seguridad y bienestar permanentes. Ayuda también en el proceso que rompe con el fenómeno de temor-tensión-dolor.

Preparación teórica: información oportuna que se brinda a la paciente para que conozca y entienda aspectos relacionados con su embarazo y parto, lo cual la ayuda a tener tranquilidad y seguridad, empoderándola respecto a los cuidados de salud y generando en ella una cultura de prevención. ${ }^{7-9}$

Dentro de los métodos y técnicas de la psicoprofilaxis obstétrica se encuentran los siguientes:

1. Técnicas de relajación: son las diferentes modalidades de lograr un estado de reposo tanto físico como mental en la madre, con la finalidad de hacerle ahorrar energía, lograr alivio de tensiones o molestias y mejorar su capacidad de concentración. Las técnicas de relajación pueden ser de manera profunda o progresiva (técnica de Jacobson y Schultz), utilizadas como técnicas básicas en sofrología, para un mejor autocontrol de las molestias.

2. Técnicas de respiración: son las diferentes modalidades de efectuar la oxigenación pulmonar, lo que a su vez mejora la oxigenación en la paciente y el bebé, dependiendo del momento y la necesidad de la paciente, con lo cual logrará también mayor comodidad y tranquilidad, facilitando la relajación y control de las molestias propias de cada fase de la gestación o trabajo de parto. Una de las técnicas más populares es el método de Lamaze: ${ }^{10-12}$

a. Respirar profundo y lento es el método más efectivo. Pero la mejor forma de hacerlo es como la paciente se sienta más cómoda. No hay reglas en cuanto al número de respiraciones por minuto, ni la forma (con la boca, nariz o produciendo ruido). La clave es realizarlo de forma consciente y no automática.

b. También se recomienda enfocarse en algo, ya sea con los ojos cerrados o abiertos, de esta forma se puede mantener un ritmo de respiraciones. c. Los cambios de posición y el masaje son estrategias que se pueden usar de forma combinada con las técnicas de respiración.

3. Técnicas de vinculación: acciones o dinámicas que buscan propiciar el lazo afectivo en la pareja respecto a la llegada del hijo(a) (Figura 1).

4. Calistenia: movimientos suaves, rítmicos y progresivos que se utilizan en especial al inicio de la preparación física de la paciente con la finalidad de que logre un estiramiento natural para una mayor comodidad, elasticidad y flexibilidad durante los movimientos en el trabajo de parto.

5. Aromaterapia: es un método alternativo mediante el uso de fragancias a través de aceites esenciales aromáticos con la finalidad de promover la salud y el bienestar del cuerpo, la mente y las emociones.

6. Esferodinamia: es una modalidad de entrenamiento físico con el uso de una esfera o pelota de plástico inflada con aire, que sirve para facilitar algunos movimientos y posturas que ayudan en la preparación física prenatal y postnatal de la madre.

7. Terapia de relajación: es un método alternativo mediante el uso de manipulaciones de los tejidos blandos del cuerpo ejercida especialmente con las manos para lograr el alivio de molestias, relajación, mejorar la circulación y oxigenación, además de favorecer un descanso adecuado y disminución de la fatiga tanto física como emocional.

8. Hidroterapia: es el uso del agua como recurso para facilitar la preparación prenatal, en especial en el área física, y que por sus características y beneficios, ayuda en los movimientos, flexibilidad y reduce el esfuerzo físico y molestias, además genera sensación de bienestar integral, entre muchos otros beneficios.

9. Terapia con música: es un método alternativo terapéutico para lograr motivación, relajación, meditación y estimulación durante la sesión de psicoprofilaxis obstétrica, con lo que se consigue un mejor estado psicofísico y optimiza el estado emocional, cognitivo y espiritual en la paciente y su pareja durante su preparación. ${ }^{5-7}$ 


\section{LO QUE CADA PACIENTE EMBARAZADA NECESITA SABER ACERCA DE LA PROFILAXIS OBSTÉTRICA}

Tanto la Organización Internacional de Lamaze como la OMS implementan seis prácticas basadas en revisiones sistemáticas buscadas en Cochrane que facilitan el parto psicoprofiláctico y que la paciente debe conocer: ${ }^{13}$

1. Dejar que el trabajo de parto inicie por sí mismo.

2. Permitir el movimiento, caminar, los cambios de posición durante el trabajo de parto.

3. Contar con apoyo continuo ya sea de familiar, amigo o doula.

4. Evitar intervenciones médicas que no sean necesarias.

5. Evitar el nacimiento desde una posición horizontal.

6. Mantener el apego del binomio.

\section{RECOMENDACIONES DE LA OMS PARA LOS CUIDADOS DURANTE EL PARTO}

Una "experiencia de parto positiva" es aquella que cumple o supera las creencias y expectativas personales y sociocul- turales previas de la mujer. Esto incluye dar a luz a un bebé sano en un ambiente seguro desde el punto de vista clínico y psicológico, así como contar con apoyo práctico y emocional continuo, es decir, estar acompañada en el momento del nacimiento y ser asistida por personal amable y con competencias técnicas adecuadas. Se basa en la premisa de que la mayoría de las mujeres desean tener un trabajo de parto y nacimiento fisiológicos y alcanzar un sentido de logro y control en la toma de decisiones, inclusive cuando se requieren o desean intervenciones médicas.

De aquí nace el objetivo de la Organización Mundial de la Salud (OMS) de crear esta directriz actualizada, integral y consolidada con el objetivo de garantizar la calidad de atención basada en la evidencia, independientemente del entorno o del nivel de atención de salud. Cabe destacar que estas recomendaciones no son específicas a ningún país o región, y que reconocen las diferencias que existen en los servicios de salud en el mundo, pero se intenta presentar un modelo global de cuidados durante el parto a pesar de la heterogeneidad de los modelos de salud y atención en los distintos países, con el fin de asegurar una atención de alta calidad en el trabajo de parto y mejores resultados centrados en la mujer (Figura 2). ${ }^{14}$

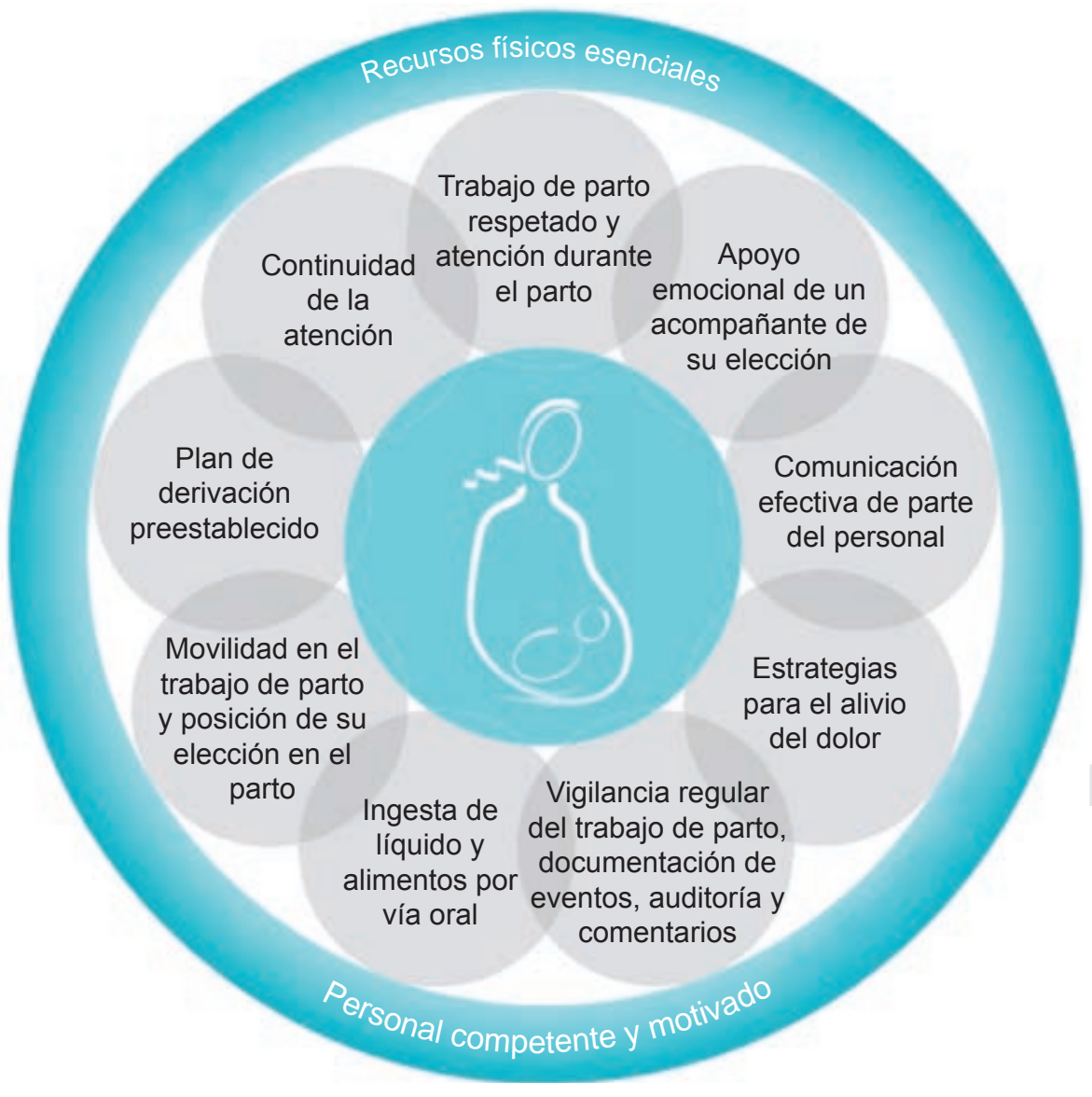

Figura 2:

Representación esquemática del modelo de cuidados durante el parto OMS. 


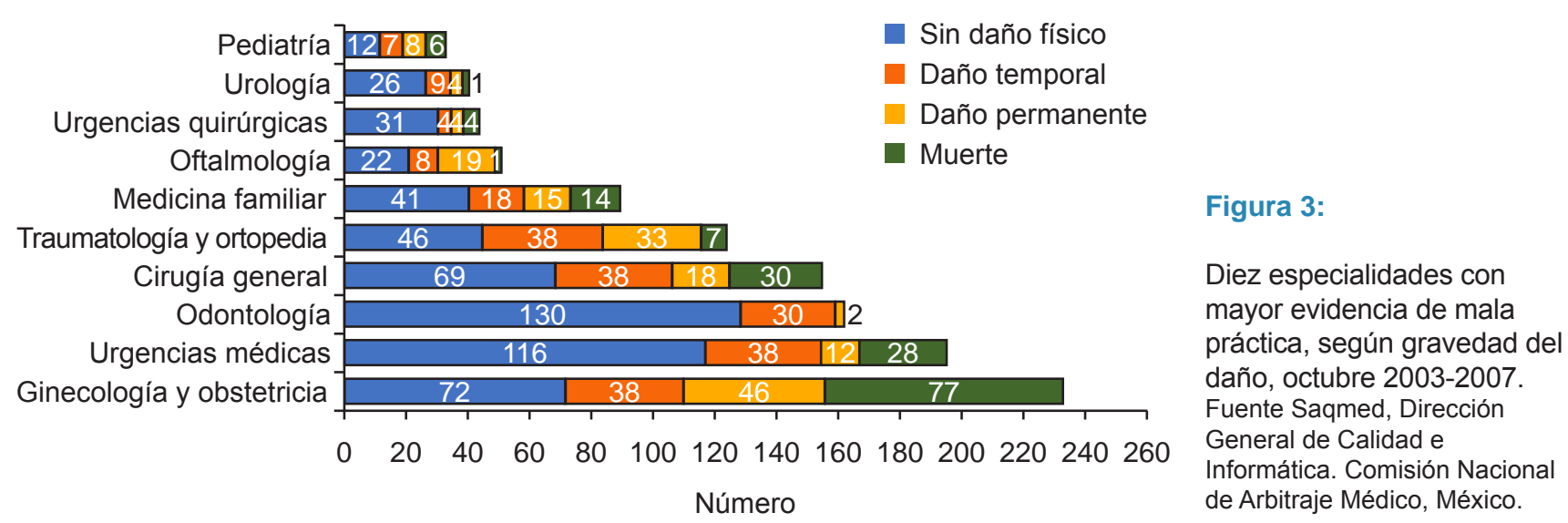

\section{REALIDAD ACTUAL: RESULTADOS DEL PARTO PSICOPROFILÁCTICO EN EL ÁMBITO MUNDIAL}

En un estudio cohorte donde se reclutaron 857 pacientes primigestas de 15 clínicas en Suiza durante el periodo de octubre 2005 a enero 2007, y se dividieron en un grupo de 486 mujeres que tuvieron un parto psicoprofiláctico y 371 que no, se concluyó que el uso de psicoprofilaxis durante el trabajo de parto se asocia con un menor riesgo de cesárea de emergencia (OR 0.57; IC de 95\%: 0.37-0.88). No se encontraron diferencias estadísticas en la duración del trabajo de parto (OR ajustado 1.32; IC de 95\% 0.95-1.83), puntaje de Apgar $<7$ a los cinco minutos (OR ajustado 0.82; IC de 95\% 0.33-2.01), analgesia epidural (OR ajustado 1.13; IC de 95\% 0.84-1.53) o experiencia de parto con miedo/ansiedad (OR ajustado 1.04; IC del 95\% 0.62-1.74). ${ }^{15,16}$

Otro estudio realizado en la universidad de Wisconsin, EUA, menciona que el miedo al parto está relacionado con una menor tolerancia al dolor de éste y un peor resultado postparto. Esta investigación, el estudio de educación prenatal sobre la reducción del estrés laboral (PEARLS, por sus siglas en inglés), es un ensayo controlado aleatorio (ECA; $n=$ 30) de un curso corto, intensivo y de 2.5 días de preparación para el parto basado en la atención plena que se ofrece como un taller de fin de semana. Las pacientes primigestas a finales del tercer trimestre del embarazo fueron asignadas al azar para asistir al curso intensivo o al curso estándar de preparación para el parto sin enfoque en la mente y el cuerpo. Al final, se demostró que la educación para el parto basada en la atención plena mejoró las evaluaciones relacionadas con el parto y el funcionamiento psicológico de las mujeres en comparación con la educación estándar. Los participantes del programa intensivo mostraron una mayor autoeficacia y conciencia corporal, síntomas de depresión más bajos después del curso que se mantuvieron durante el seguimiento postparto y una tendencia hacia una tasa más baja de uso de analgesia con opioides en el trabajo de parto. ${ }^{17,18}$
A pesar del uso extenso de la psicoprofilaxis y de los beneficios demostrados, existe poca evidencia científica de la misma, por lo que aún queda mucho por investigar, sobre todo acerca de los resultados perinatales y neonatales. ${ }^{19,20}$

\section{REALIDAD ACTUAL DE LA PSICOPROFILAXIS OBSTÉTRICA EN MÉXICO}

A través de herramientas interculturales de escucha, se dio a conocer que había muchas mujeres en regiones urbanas y suburbanas que luchaban por un modelo humanizado, en las que ellas fueran sujetos y no objetos del parto. Esto llevó a conocer e investigar la temática de la violencia obstétrica y se planteó la necesidad de enriquecer el modelo convencional de atención, con aportes del modelo tradicional, que coincidieran con las recomendaciones de la OMS.

En la revisión Cochrane titulada Apoyo continuo para las mujeres durante el parto, que forma parte de la Biblioteca de Salud Reproductiva de la OMS, se señala que es frecuente ver en hospitales de países de medianos ingresos, en especial en América Latina, a mujeres en trabajo de parto sin apoyo y bajo un modelo de atención medicalizado y deshumanizado, el cual prevalece en zonas urbanas, donde reside más de $70 \%$ de la población. En México, más de $90 \%$ de las mujeres que viven en las grandes ciudades tienen su parto en hospitales. Los índices elevados de episiotomías y cesáreas son indicadores del elevado nivel de medicalización de la atención materna en estos lugares, el cual se describe en la literatura como aquél que promueve el uso de intervenciones innecesarias, descuida las necesidades emocionales de las embarazadas y contribuye al incremento del costo global de los servicios médicos.

A partir de los registros de la Comisión Nacional de Arbitraje Médico, se comparan las quejas por mala práctica entre diversas especialidades médicas, y se observa que 
la atención gíneco-obstétrica es la que presenta el mayor número de casos y poco más de la mitad de ellos se clasificó como daño permanente y muerte. La insatisfacción de las usuarias está relacionada con la violencia obstétrica o perinatal, problemática nacional ampliamente conocida, de la cual se cuenta con estudios cualitativos, es por eso que surge una preocupación por mejorar la calidad de atención en nuestras pacientes embarazadas (Figura 3). ${ }^{21}$

En enero de 2005, se publicó en el Diario Oficial de la Federación la resolución por la que se modificó la Norma Oficial Mexicana NOM-007-SSA2-1993 (NOM 007) que busca disminuir los daños obstétricos y los riesgos para la salud de las mujeres y de sus hijos en el marco de la atención del embarazo, el parto y el puerperio. Esta norma pone énfasis en la realización de actividades preventivas de riesgos durante el embarazo, así como la racionalización de ciertas prácticas que se llevan a cabo de forma rutinaria y que aumentan los riesgos innecesarios. Asimismo, se plantea la necesidad de fortalecer la calidad y calidez de los servicios de atención médica durante el embarazo, el parto y el puerperio. ${ }^{22}$ A partir de esto, la Secretaría de Salud del país implementa una guía que pretende facilitar a los servicios de salud y el personal directivo y operativo las condiciones para la implantación del modelo de atención a las mujeres en el parto con enfoque humanizado, intercultural y seguro, así como brindar toda la información para modificar el paradigma de atención del embarazo y del recién nacido, tomando en cuenta las recomendaciones de la OMS, las evidencias científicas, los derechos humanos y el marco normativo nacional. También surge la "Unidad de Posada de Nacimiento" como resultado de la necesidad de otorgar a la mujer una atención que tome en cuenta el enfoque basado en evidencias científicas y que responda a las recomendaciones de la OMS. La Unidad de Posada de Nacimiento se define de esta manera como una ampliación de un hospital destinado a ofrecer hospedaje a embarazadas y puérperas (con camas no censables), atención del parto normal bajo el modelo con enfoque humanizado, intercultural y seguro, vigilancia del puerperio a las mujeres que lo requieran, así como albergue a sus hijos y/o acompañantes. Está inspirada en las posadas AME (Albergue de la Mujer Embarazada) y las casas de la mujer de la Comisión Nacional para el Desarrollo de los Pueblos Indígenas (CDI) que han incorporado la atención de partos, así como por los resultados de los módulos de medicina y partería tradicional de los hospitales integrales del estado de Puebla. Por su impacto en la erradicación de la muerte materna, el Hospital Integral de Cuetzalan, Puebla, se hizo merecedor al Premio Nacional de Calidad en Salud 2007 con este modelo. ${ }^{23,24}$

En el ámbito privado, se forma la Asociación Nacional de Instructoras en Psicoprofilaxis Perinatal en 1987 con el firme compromiso de ayudar a la mujer mexicana y al parto humanizado en México, y a su vez se ha esforzado por contar con contactos importantes para el desarrollo de la educadora perinatal, por lo que se han llevado a cabo convenios y alianzas con hospitales y asociaciones como Bité Médica, Hospital Central Militar, INPer, Grupo Ángeles, entre otros. Por ejemplo, en el Hospital Ángeles Lomas se ofrece el curso de psicoprofilaxis entre el cuarto y quinto mes de embarazo, con sesiones diarias que duran entre tres y cuatro horas. Del total de partos mensuales que se presentan en este hospital, entre 18 a $25 \%$ son psicoprofilácticos, pero aún queda más por discernir, sobre todo en cuanto a los resultados perinatales y neonatales a corto y largo plazo, y compararlos con los resultados publicados en la literatura internacional. ${ }^{25}$

\section{CONCLUSIONES}

Las mujeres que realizan el curso de psicoprofilaxis perinatal participan en forma activa durante el trabajo de parto, controlan el temor, dolor y aplican las técnicas no farmacológicas, logrando con esto acortar el tiempo de trabajo de parto y obtienen conocimientos acerca del proceso, lo que rompe con esta cadena de miedo-ansiedad-dolor que se genera. La psicoprofilaxis obstétrica es una alternativa, y el propósito principal de este método es completar esta educación, y a su vez las mujeres pierden la actitud pasiva que adoptan cuando llega el parto, saben lo que va a suceder y aprenden a adaptarse y a controlar las modificaciones que ocurren en su organismo durante el trabajo de parto. Otro de los objetivos del parto psicoprofiláctico, "humanizado", "respetado" es darle un enfoque basado en los derechos humanos, donde la premisa es que sea necesario empoderar a la mujer para que reivindique sus derechos, y las transforme en agentes activas facultadas para participar en las decisiones que afectan a su salud sexual y reproductiva, tomando como base las recomendaciones de la OMS y los convenios internacionales basados en evidencia. Aún queda mucho por hacer para mejorar los distintos niveles de atención en nuestro país, sobre todo dentro de las instituciones públicas y también queda mucho por investigar en cuanto a los resultados perinatales, y de esta forma conocer las mejoras que se pueden aplicar.

\section{REFERENCIAS}

1. Lothian JA. Safe, healthy birth: what every pregnant woman needs to know. J Perinat Educ. 2009; 18 (3): 48-54.

2. Lowe NK. The nature of labor pain. Am J Obstet Gynecol. 2002; 186 (5 Suppl Nature): S16-S24.

3. Gazca JN. Parto indoloro psicoprofiláctico. 20 observaciones personales. Ginecol Obstet Mex. 2014; 82 (3): 210-222. 
4. Karmel M. Thank You, Dr Lamaze. London: Pinter \& Martin Ltd.; 2005.

5. Lamaze International. Lamaze healthy birth practices. 2009. Available in: http://www.lamaze.org/ExpectantParents/HealthyBirthPractices/ tabid/251/Default.aspx

6. Lothian JA, DeVries C. The official Lamaze guide: Giving birth with confidence. 2nd ed. Minnetonka, MN: Meadowbrook Press; 2010.

7. Morales AS, Guibovich MA, Yábar PM. Psicoprofilaxis obstétrica: actualización, definiciones y conceptos. Horiz Med. 2014; 14 (4): 53-57.

8. Caton D, Corry MP, Frigoletto FD, Hopkins DP, Lieberman E, Mayberry $\mathrm{L}$ et al. The nature and management of labor pain: executive summary. Am J Obstet Gynecol. 2002; 186 (5S): S1-15.

9. Waldenstrom $U$, Irestedt L. Obstetric pain relief and its association with remembrance of labor pain at two months and one year after birth. J Psychosom Obstet Gynaecol. 2006; 27 (3): 147-156.

10. Physical Activity and Exercise During Pregnancy and the Postpartum Period: ACOG Committee Opinion, Number 804. Obstet Gynecol. 2020; 135 (4): e178-e188.

11. Nichols FH. Paced breathing techniques. In: Nichols FH, Humenick SS, editors. Childbirth education: practice, research and theory. 2nd ed. Philadelphia, PA: W. B. Saunders; 2000. pp. 271-283.

12. Smith CA, Collins CT, Cyna AM, Crowther CA. Complementary and alternative therapies for pain management in labour. Cochrane Database Syst Rev. 2006; 2006 (4): CD003521.

13. Simkin P, Bolding A. Update on nonpharmacologic approaches to relieve labor pain and prevent suffering. J Midwifery Womens Health. 2004; 49 (6): 489-504.

14. Recomendaciones de la OMS sobre atención prenatal para una experiencia positiva del embarazo. Washington, D.C.: Organización Panamericana de la Salud; 2018.

15. Bergstrom M, Kieler H, Waldenstrom U. Psychoprophylaxis during labor: associations with labor-related outcomes and experience of childbirth. Acta Obstet Gynecol Scand. 2010; 89 (6): 794-800.
16. Bergstrom $M$, Kieler $\mathrm{H}$, Waldenstrom $\mathrm{U}$. Effects of natural childbirth preparation versus standard antenatal education on epidural rates, experience of childbirth and parental stress in mothers and fathers: a randomised controlled multicentre trial. BJOG. 2009; 116 (9): 1167-1176.

17. Bardacke N. Mindful birthing: training the mind, body, and heart for childbirth and beyond. New York: HarperOne; 2012.

18. Byrne J, Hauck Y, Fisher C, Bayes S, Schutze R. Effectiveness of a mindfulness-based childbirth education pilot study on maternal selfefficacy and fear of childbirth. J Midwifery Womens Health. 2014; 59 (2): 192-197.

19. Duncan LG, Cohn MA, Chao MT, Cook JG, Riccobono J, Bardacke $\mathrm{N}$. Benefits of preparing for childbirth with mindfulness training: a randomized controlled trial with active comparison. BMC Pregnancy Childbirth. 2017; 17 (1): 140.

20. Fabian HM, Radestad IJ, Waldenstrom U. Characteristics of Swedish women who do not attend childbirth and parenthood education classes during pregnancy. Midwifery. 2004; 20 (3): 226-235.

21. Almaguer JA, García H, Vargas V. La violencia obstétrica. Una forma de patriarcado en las instituciones de salud. Género y Salud en Cifras. 2010; 8 (3): 3-20.

22. Norma Oficial Mexicana PROY-NOM-007-SSA2-2010. Establece los requisitos para la atención de la mujer durante el embarazo, parto y puerperio, y del recién nacido. Publicado en el DOF el 05/11/12.

23. Secretaría de Salud. Guía de implantación del modelo de atención a las mujeres durante el embarazo, parto y puerperio. Enfoque humanizado, intercultural y seguro. 2012.

24. "Guía Metodológica para la Estructuración de Planes y Programas de la Formación de la Técnica en Partería", realizado para la Comisión Interinstitucional para la Formación de Recursos Humanos en Salud (CIFRHS), 2011 y 2012.

25. Asociación Nacional de Instructoras en Psicoprofilaxis Perinatal. Disponible es: http://grupocoras.com.mx/que-es-la-anipp-y-porquees-importante-una-instructoracertificada/ 